\title{
A general model based on artificial neural network for estimating the viscosities of oxygenated fuels
}

Xiangyang Liu*, Feng Yang, Jianchun Chu, Chenyang Zhu, Maogang He, Ying Zhang

Key Laboratory of Thermal Fluid Science and Engineering of MOE, School of Energy and Power

Engineering, Xi'an Jiaotong University, Xi'an 710049, China

*Corresponding author. Tel:+86-29-8266-3863; Fax:+86-29-8266-3863

E-mail address: 1xyyang@mail.xjtu.edu.cn 
Table S1 The information of chemicals stated in the literature

\begin{tabular}{|c|c|c|c|c|c|}
\hline Ref. & Component & $\begin{array}{c}\text { CAS } \\
\text { Reg.No. }\end{array}$ & Supplier & $\begin{array}{c}\text { Mass } \\
\text { fraction } \\
\text { purity/\% }\end{array}$ & $\begin{array}{l}\text { Purification } \\
\text { method }\end{array}$ \\
\hline 14 & Ethyl heptanoate & $106-30-9$ & Sigma-Aldrich & 99 & - \\
\hline 14 & Ethyl octanoate & 106-32-1 & Sigma-Aldrich & 99 & - \\
\hline 15 & Methyl laurate & $111-82-0$ & Sigma-Aldrich & 99 & - \\
\hline 15 & Ethyl laurate & $106-33-2$ & Sigma-Aldrich & 99 & - \\
\hline 16 & Methyl caprate & $110-42-9$ & Sigma-Aldrich & 99 & - \\
\hline 16 & Ethyl caprate & $110-38-3$ & Sigma-Aldrich & 99 & - \\
\hline 17 & Ethyl myristate & 124-06-1 & Sigma-Aldrich & 99 & - \\
\hline 17 & Methyl myristate & $124-10-7$ & Sigma-Aldrich & 99 & - \\
\hline 18 & Methanol & $67-56-1$ & BDH Chemicals Ltd. & 99.8 & - \\
\hline 18 & Ethanol & $64-17-5$ & BDH Chemicals Ltd. & 99.8 & - \\
\hline 18 & 1-Propanol & $71-23-8$ & BDH Chemicals Ltd. & 99.8 & - \\
\hline 19 & Methanol & $67-56-1$ & $\begin{array}{c}\text { Wako Pure Chemical } \\
\text { Industries Ltd. }\end{array}$ & 99.5 & - \\
\hline 19 & Ethanol & $64-17-5$ & $\begin{array}{c}\text { Wako Pure Chemical } \\
\text { Industries Ltd. }\end{array}$ & 99.5 & - \\
\hline 19 & 1-Propanol & $71-23-8$ & $\begin{array}{c}\text { Wako Pure Chemical } \\
\text { Industries Ltd. }\end{array}$ & 99.5 & - \\
\hline 19 & 2-Propanol & $67-63-0$ & $\begin{array}{c}\text { Wako Pure Chemical } \\
\text { Industries Ltd. }\end{array}$ & 99.5 & - \\
\hline 19 & 2-Methyl-2-propanol & $75-65-0$ & $\begin{array}{c}\text { Wako Pure Chemical } \\
\text { Industries Ltd. }\end{array}$ & 99.5 & - \\
\hline 37 & Methanol & $67-56-1$ & $\begin{array}{c}\text { Wako Pure Chemical } \\
\text { Industries Ltd. }\end{array}$ & 99.9 & - \\
\hline 37 & 2-Methyl-2-Propanol & $75-65-0$ & $\begin{array}{c}\text { Wako Pure Chemical } \\
\text { Industries Ltd. }\end{array}$ & 99.9 & - \\
\hline 38 & Ethanol & $64-17-5$ & Riedel-de-Haën & 99.8 & - \\
\hline 39 & 2-Propanol & 67-63-0 & Sigma-Aldrich & 99.5 & \\
\hline 40 & 1-Pentanol & 71-41-0 & Sigma-Aldrich & 99 & - \\
\hline 40 & 3-Pentanol & $584-02-1$ & Sigma-Aldrich & 98 & - \\
\hline 40 & 1-Nonanol & 143-08-8 & Sigma-Aldrich & 98 & - \\
\hline 40 & Vinyl acetate & $108-05-4$ & Merck & 99 & - \\
\hline 41 & Diethyl adipate & $141-28-6$ & $\begin{array}{c}\text { Sinopharm Chemical } \\
\text { Reagent Co.Ltd. }\end{array}$ & 99 & - \\
\hline 43 & Diethyl carbonate & $105-58-8$ & Fluka & 99.5 & Drying \\
\hline 43 & $\begin{array}{l}\text { Tetraethylene glycol } \\
\text { dimethyl ether }\end{array}$ & $143-24-8$ & Sigma-Aldrich & 99 & Drying \\
\hline 43 & Dimethyl carbonate & 616-38-6 & Fluka & 99 & Drying \\
\hline 43 & Triethylene glycol & $112-49-2$ & Sigma-Aldrich & 99 & Drying \\
\hline
\end{tabular}




\begin{tabular}{|c|c|c|c|c|c|}
\hline & dimethyl ether & & & & \\
\hline 42 & Dimethyl carbonate & $616-38-6$ & $\begin{array}{c}\text { Hebei Newchaoyang } \\
\text { Chemical Stock Co., Ltd. }\end{array}$ & 99.7 & - \\
\hline 44 & Diethyl carbonate & $105-58-8$ & $\begin{array}{c}\text { Hebei Newchaoyang } \\
\text { Chemical Stock Co., Ltd. }\end{array}$ & 99.7 & - \\
\hline 45 & Methyl laurate & $111-82-0$ & Sigma-Aldrich & 99.5 & \\
\hline 45 & Ethyl laurate & 106-33-2 & TCI & 99 & - \\
\hline 46 & Diethyl ether & $60-29-7$ & $\begin{array}{l}\text { Tianjin Dongliqu Tianda } \\
\text { chemical Reagent Factory }\end{array}$ & 99.5 & - \\
\hline 47 & Diisopropyl ether & $108-20-3$ & Alfa Aesar & 98 & - \\
\hline 47 & Dibutyl ether & $142-96-1$ & Alfa Aesar & 99 & - \\
\hline 48 & Dimethoxymethane & $109-87-5$ & $\begin{array}{c}\text { Anhui Jixi Sanming } \\
\text { Industry of Fine Chemicals } \\
\text { Co., Ltd }\end{array}$ & 99.9 & - \\
\hline 48 & $\begin{array}{l}\text { Ethylene glycol } \\
\text { dimethyl ether }\end{array}$ & $110-71-4$ & $\begin{array}{c}\text { Anhui Jixi Sanming } \\
\text { Industry of Fine Chemicals } \\
\text { Co., Ltd }\end{array}$ & 99.9 & - \\
\hline 49 & $\begin{array}{l}\text { Diethylene glycol } \\
\text { dimethyl ether }\end{array}$ & $111-96-6$ & $\begin{array}{c}\text { Anhui Jixi Sanming } \\
\text { Industry of Fine Chemicals } \\
\text { Co., Ltd }\end{array}$ & 99.5 & - \\
\hline 50 & $\begin{array}{l}\text { Monoethylene glycol } \\
\text { methyl ether }\end{array}$ & $109-86-4$ & Sigma-Aldrich & 99.3 & - \\
\hline 50 & $\begin{array}{l}\text { Monoethylene glycol } \\
\text { iso-propyl ether }\end{array}$ & $109-59-1$ & Sigma-Aldrich & 99 & - \\
\hline 50 & $\begin{array}{c}\text { Monoethylene glycol } \\
\text { ethyl ether }\end{array}$ & $110-80-5$ & Riedel de Haën & 99 & - \\
\hline
\end{tabular}

